\title{
Performance Estimation of Sustainable Smart Farming with Blockchain Technology
}

\author{
Dr. D. Sivaganesan, \\ Professor, \\ Department of Computer Engineering, \\ PSG Institute of Technology and Applied Research, \\ Coimbatore, India. \\ Email: sivaganesan@psgitech.ac.in
}

\begin{abstract}
Sustainable smart agriculture with increase in signal to interference or signal to noise ratio (SIR/SNR) for selection of best relay is discussed in a wireless blockchain based network. The overall communication throughput (OCT), power splitting relaying (PSR), time switching relaying (TSR) and transmission success rate (TRS) are also derived during the selection of best relay performance with and without interference. The performance of OCT, PSR, TSR and TRS increases with the increase in the number of potential relay nodes as seen in the results of derivation. The accuracy of the theoretical values are validated by numerical simulations.
\end{abstract}

Keywords: Sustainable farming, Time switching relay, Blockchain, Relaying, Wireless sensor network, Communication

\section{Introduction}

Accounting and storage purposes of next generation are satisfied by means of the promising next generation blockchain technology [1]. The data security is enhanced in blockchain due to the decentralization characteristics which makes it challenging to corrupt the stored information[2]. When anonymous participants are involved, the transactions may be traced in a reliable manner using blockchain technology. In case of food safety and quality related issues, optimal solutions and transparency are provided by this technology [3]. In scenarios like unmanned farm and wastelands, where effective management of transfer and payment may not be performed by a central authority, blockchain enables distributed management. In sustainable smart farming, wireless networks enabled with blockchain technology that make use of Decode and Forward (DF) has been investigated largely [4]. The Nakagami-m, Rice and Rayleigh channels that are commonly used impose various challenges while modeling the complex 
agricultural environment and channel conditions [5]. It is also impossible to omit interferences in certain blockchain-enabled wireless networks due to the large number of smart devices involved. Also, unclear DF relay performance for sustainable smart farming using wireless networks equipped with blockchain technology is challenging [6].

External charging mechanism is required in wireless sensor networks as the sensor nodes and relays have limited power storage capabilities [7]. This problem can be overcome using energy harvesting techniques. In wireless networks equipped with blockchain technology for sustainable smart farming, the relay based systems are discussed scarcely in the existing literature $[8,9]$. The overall communication throughput (OCT), power splitting relaying (PSR), time switching relaying (TSR) and transmission success rate (TRS), which are the most significant metrics in wireless networks equipped with blockchain technology are investigated [10]. In networks with blockchain, the data transmission, throughput and success rate are evaluated. In case of multiple potential relay nodes scenarios, the optimal selection of relay has been proposed. The transmission channel model considered here has a general a-m channel distribution. The performance of elimination of interference signals at full function and relay nodes and their adverse effects other than during ideal transmission are also investigated [11]. The OCT, PSR, TSR and TRS closed-for expressions are derived while their performance metrics and network parameter relationship is analyzed.

\section{Related Works}

The productivity and sustainability of the process of agricultural production has improved largely with smart sensors, intelligent agricultural machinery and other smart devices with the rapid evolution in information and communication technologies [12]. Further advancements in smart farming are proposed with more intelligent systems and models. Tracking of quality assurance, data integrity, record management and so on are considered while integrating blockchain with smart farming system due to the features of blockchain technology [13]. This has attracted the attention of several industrial and academic researchers over the past few years. Sensors and low power devices in the Internet of Things (IoT) networks form the functional structure of blockchain based smart farming system [14]. The system is maintained in an ecological, open, self-organized and trusted manner by deploying blockchain based on IoT for enhancing the food traceability. Certification based on blockchain technology is 
implemented on a real time scenario for obtaining assurance tracking on the grain quality. Across the supply chain of agricultural produce, traceability and tracking of soybean is performed efficiently by means of business transactions using Ethereum blockchain based smart contracts [15].

It is challenging to meet the growing requirements for direct communication among blockchain nodes and smart devices due to the poor communication models and conventional cellular systems existing in the rural regions leading to poor coverage [16]. The smart farming system must be integrated with flexible transmission schemes to overcome this issue. Relay assisted transmission is a feasible solution where the transmission quality and transmission distance may be enhanced by forwarding the received signals using a relay. Compress and forward, decode and forward, amplify and forward and several other relay strategies are available for this purpose [17]. The original mode of transmission is compatible with the DF relay model which makes it an easy deployment solution. A linear complexity based multicast capacity is approximated by an algorithm while analyzing the DF relay system with two hop in terms of its multicast capacity [18]. The partial coding of Cover-El Gamal and Marton coding is combined for providing the relay channels of three nodes with a distributed DF scheme. Under the Nakagami-m fading channels, the cognitive DF relay networks and their performance in terms of secrecy is analyzed. For a DF relay system with multicarrier, full-duplex, dual hope features, the power allocation model has been investigated in which the group-wise and carrierwide DF models are used [19].

\section{Proposed Work}

The proposed wireless networks equipped with blockchain technology for sustainable smart farming is as show in figure 1. The fully functional nodes (FFNs), relay nodes (RNs) and transaction nodes (TNs) are the different nodes available in the network. Solar energy charging pile, smart water meter, smart electric meter and other such smart devices with the ability of blockchain request and that are low-power and low-cost sensor nodes are generally categorized under the transaction nodes. The general data and amount used is included in the raised transaction when the farming application makes use of the TNs. Before building a new block for transaction, it is essential to transmit the transaction that is raised by a wireless link to an 
FFN. This leads to anomalies for the TNs to operate as normal blockchain nodes causing poor correlation with other nodes and computational capabilities.

Impracticability and ineffectiveness is observed when the TNs undertake direct transmission to the FFNs due to the low transmit power of TNs. The temporarily inactive TNs or the network's pre-arranged RNs may be used for overcoming this drawback in the implementation of sustainable smart farming with blockchain technology. The transmission of transaction to RNs from TNs will be performed with the help of RNs and further, it is transmitted to the FFNs from RNs. Other than the required transaction signals, interference signals may be received by the FFN and TN receiver if multiple transactions occur simultaneously. Wired links are used for notifying the other FFNs on the successful reception of transaction by the FFN in order to confirm the reception and storage in blocks. Data received by the RNs are decoded and transmitted to the FFN after modulation based on the DF mode of the RN and its deployment flexibility. The system performance may be enhanced by easy deployment of RNs. The a-m distribution is used for modeling the wireless channels in a complex agricultural environment. The Nakagami-m, Rice and Rayleigh channel are compared to the proposed model which is much more generalized.

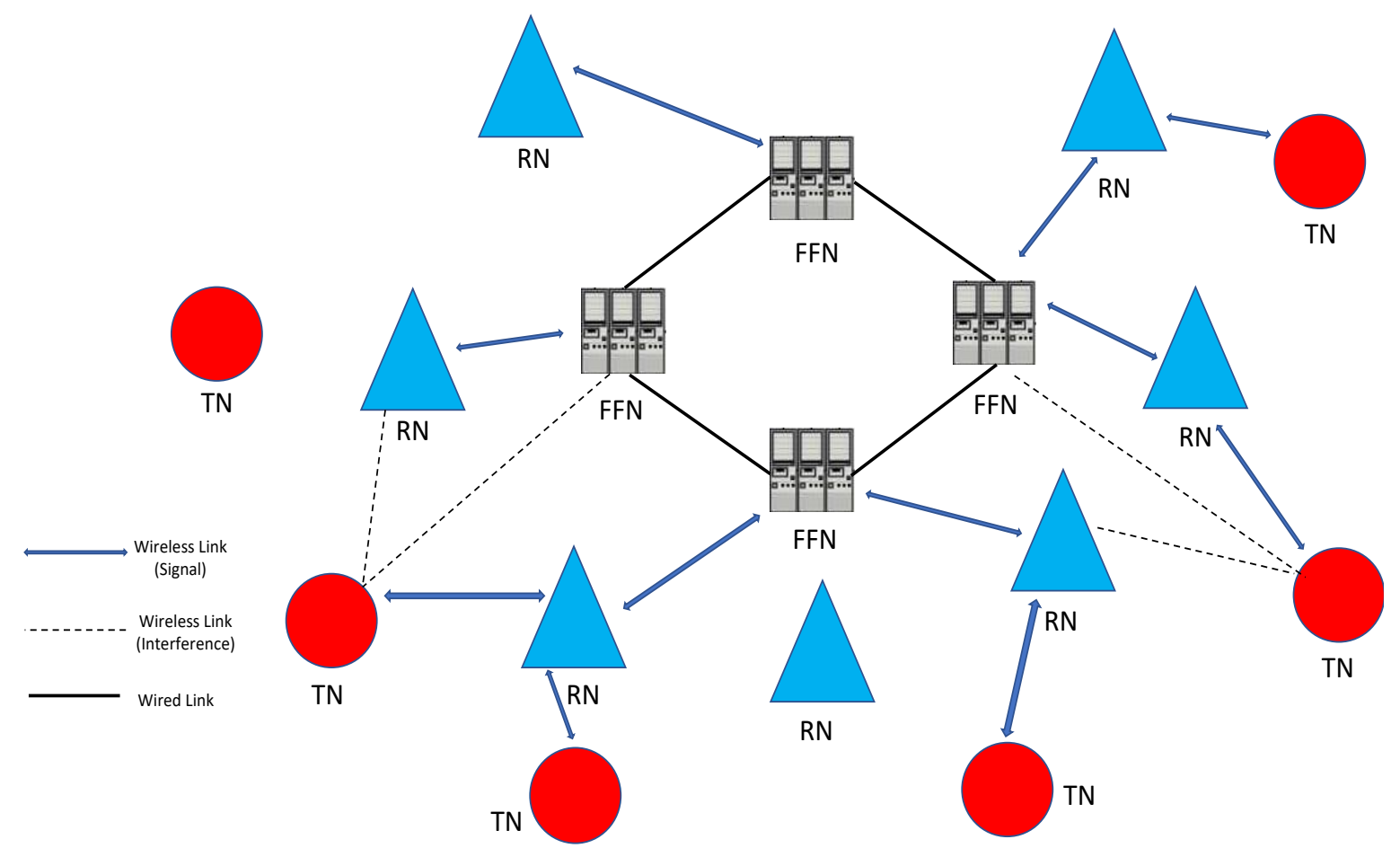

Fig. 1. Proposed wireless networks architecture for sustainable smart farming 
The $\mathrm{RN}$ receive signal is given by

$$
\mathrm{RS}_{\mathrm{RN}}=\sqrt{P_{T N} C_{1} T S_{T N}}+\mathrm{N}_{\mathrm{RN}}
$$

When interference does not exist. Here, $\mathrm{P}_{\mathrm{TN}}$ is the power transmitted by the TN involved, $\mathrm{N}_{\mathrm{RN}}$ is the noise received at $\mathrm{RN}, \mathrm{TS}_{\mathrm{RN}}$ is the signal transmitted by the corresponding $\mathrm{TN}$ and $\mathrm{C}_{1}$ is the channel between TN and RN. Further, the receive signal for FFN is represented by the following expression

$$
\mathrm{RS}_{\mathrm{FFN}}=\sqrt{P_{R N} C_{2} T P_{R N}}+\mathrm{N}_{\mathrm{FFN}}
$$

Where, the noise received at FFN is represented by $\mathrm{N}_{F F N}$, the channel between FFN and RN is given by $\mathrm{C}_{2}$, the RN's transmission power is represented by $\mathrm{TP}_{\mathrm{RN}}$ and $\mathrm{RS}_{\mathrm{FFN}}$ is the signal received at the FFN. The normalized values of $\mathrm{TS}_{\mathrm{TN}}$ and $\mathrm{TP}_{\mathrm{RN}}$ helps in deriving the $\mathrm{SNR}$ value of $\mathrm{RN}$ and FFN using the following expressions.

$$
\begin{gathered}
S N R_{R N}=\frac{P_{T N\left|C_{1}\right|^{2}}}{\delta_{R N}^{2}} \text {----- } \\
S N R_{F F N}=\frac{P_{R N\left|C_{2}\right|^{2}}}{\delta_{F F N}^{2}}-\text {---- }
\end{gathered}
$$

Where the signal to noise ratio at $\mathrm{RN}$ and FFN are represented as $\mathrm{SNR}_{\mathrm{RN}}$ and $\mathrm{SNR}_{\mathrm{FFN}}$ respectively. The noise variance at RN and FFN are represented as $\delta_{R N}^{2}$ and $\delta_{F F N}^{2}$ respectively. Considering the threshold value $t$,

$$
\begin{gathered}
\mathrm{TRS}=\operatorname{Pr}\{\operatorname{SINR}>\mathrm{t}\}------(5) \\
\mathrm{OCT}=\operatorname{LMPr}\{\mathrm{SINR}>\mathrm{t}\}------(6)
\end{gathered}
$$

Where $\mathrm{M}$ represents the total raised transactions and $\mathrm{L}$ is the transaction size. 


\section{Results and Discussion}

The OCT, PSR, TSR and TRS values are estimated for performance evaluation of the proposed wireless networks equipped with blockchain technology for sustainable smart farming. For the transmission of blockchain transaction, the overall communication throughput required is provided by the OCT while the successful transmission probability is provided by the TRS. The threshold value may be used for obtaining the OCT and TRS values. The various performance parameters are simulated numerically to validate and analyze the performance of the model. Figure 2 represents the cumulative distribution function of the signal to noise ratio in the absence of interference. This value is also estimated when interference is present under various settings of channel distribution.

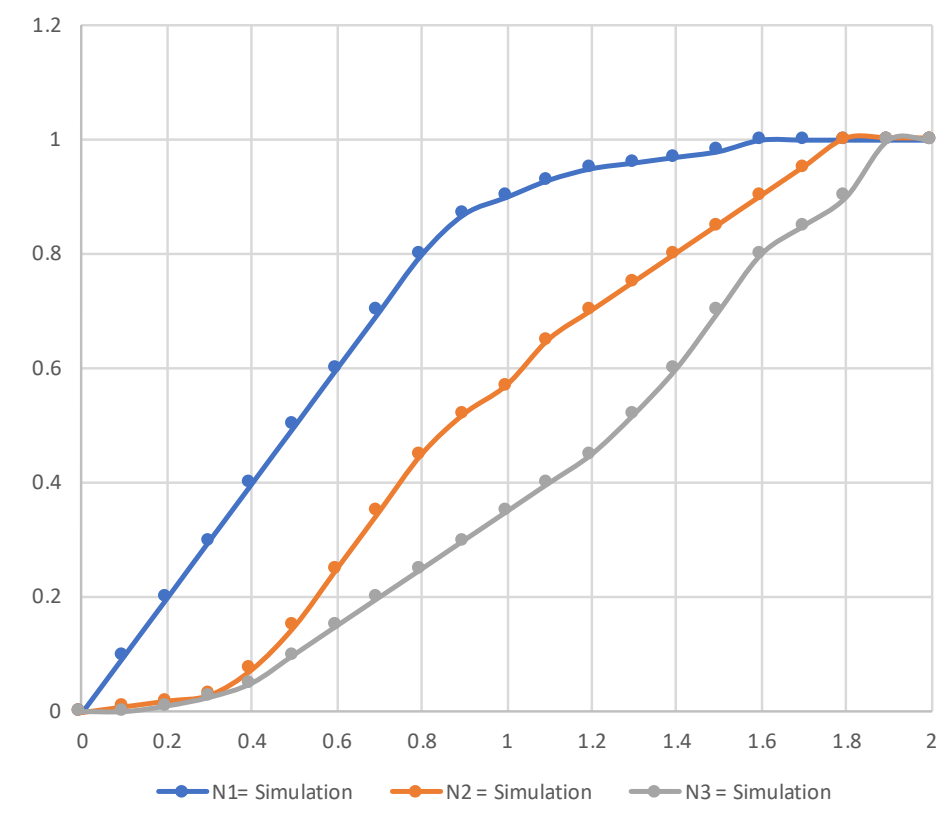

Fig. 2. Signal to noise ratio in the absence of interference

The performance of DF relay networks in terms of TRS performance in the absence of interference is also simulated under various channel distribution settings and $\mathrm{RN}$ count. There is a significant increase in the performance of TRS on adopting of the best relay selection model. There is an optimal match between the simulation and analytical curves. Figure 3 provides the comparison of OCT in the presence of interference in a DF relay network enabled with blockchain technology under various RNs. A much faster and maximum OCT may be 
reached with a large number of RNs as the SNR transmission increases as represented in this image. Signal forwarding may be performed with multiple RNs. Relay selection requires SIR maximization.

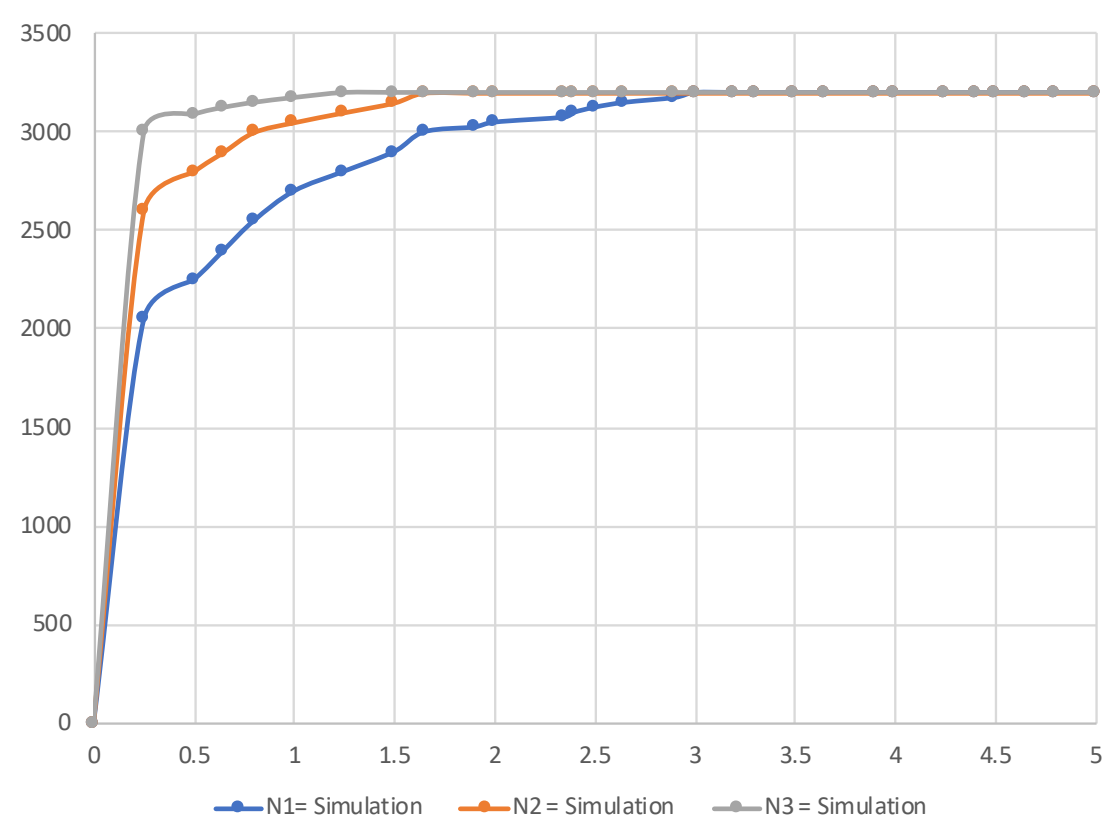

Fig. 3. OCT in the presence of interference

Figure 4 represents the signal to noise ratio in the presence of interference. Transmission may be performed by selecting the $\mathrm{RN}$ with best potential when several $\mathrm{RNs}$ are available for relaying the signal. The OCT, PSR, TSR and TRS factors helps in obtaining the SINR value. SNR and SNIR are equal to each other in the absence of interference at the RNs. The maximum end to end SNR may be used as the criterion for selection of the optimal RN. 


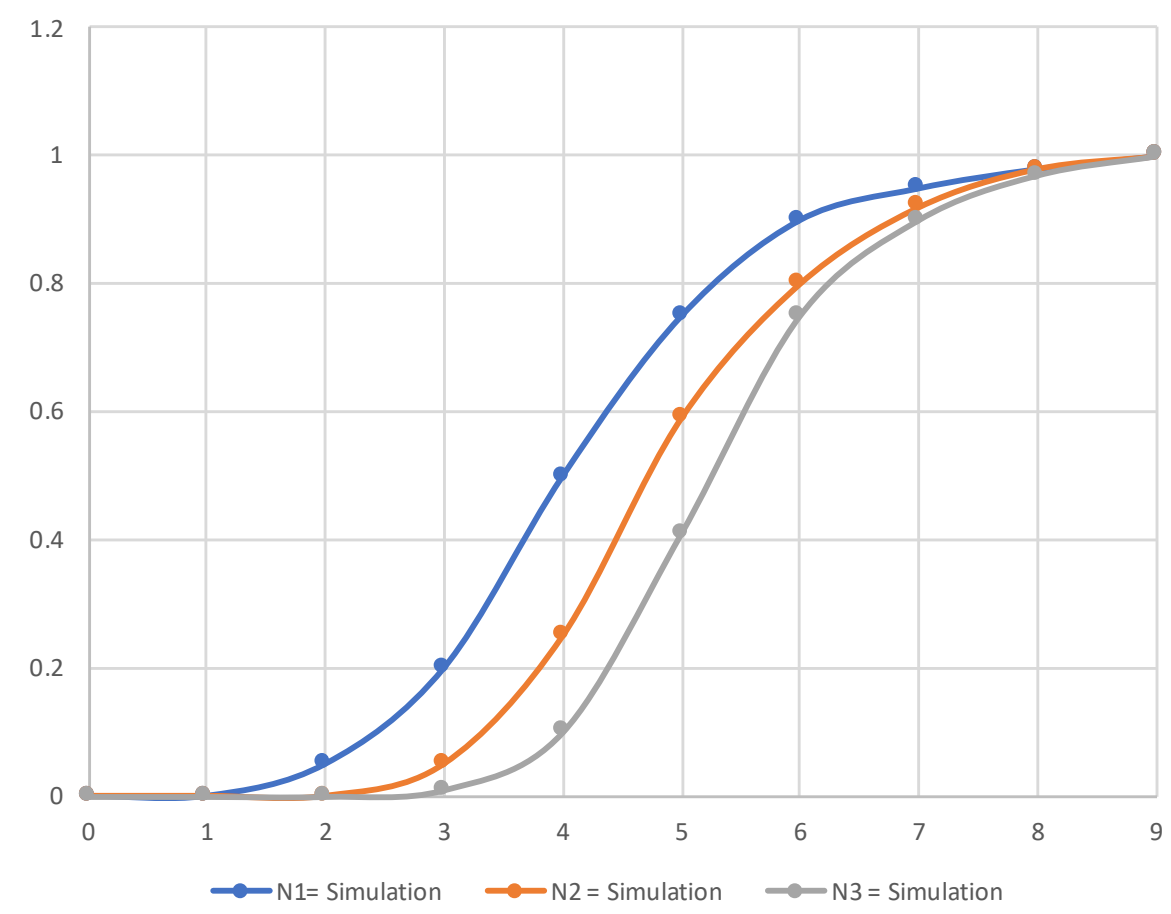

Fig. 4. Signal to noise ratio in the presence of interference

\section{Conclusion}

Sustainable smart farming system is proposed in this paper that operates on a wireless network enabled with blockchain technology whose performance is enhanced by maximization of SIR or SNR values for selection of an optimal relay on an end-to-end basis. The OCT, PSR, TSR and TRS closed-for expressions are derived while their performance metrics and network parameter relationship is analyzed and modelled mathematically. The results show a proportional increase in the performance of OCT and TRS with the increase in the relay node count. The accuracy of the theoretical concept is verified with numerical simulations. Future work is directed towards real-time analysis of the values in a controlled smart farming environment. 


\section{References}

[1] Akram, S. V., Malik, P. K., Singh, R., Anita, G., \& Tanwar, S. (2020). Adoption of blockchain technology in various realms: Opportunities and challenges. Security and Privacy, 3(5), e109.

[2] Mugunthan, S., and T. Vijayakumar. "Review on IoT based smart grid architecture implementations." j Electric Eng Autom 1, no. 1 (2019): 12-20.

[3] Alam, M. A., Ahad, A., Zafar, S., \& Tripathi, G. (2020). A NEOTERIC SMART AND SUSTAINABLE FARMING ENVIRONMENT INCORPORATING BLOCKCHAINBASED ARTIFICIAL INTELLIGENCE APPROACH. Cryptocurrencies and Blockchain Technology Applications, 197-213.

[4] Islam, N., Rashid, M. M., Pasandideh, F., Ray, B., Moore, S., \& Kadel, R. (2021). A review of applications and communication technologies for Internet of Things (IoT) and Unmanned Aerial Vehicle (UAV) based sustainable smart farming. Sustainability, 13(4), 1821.

[5] Chen, Joy Iong-Zong, and Jen-Ting Chang. "Route Choice Behaviour Modeling using IoT Integrated Artificial Intelligence." Journal of Artificial Intelligence 2, no. 04 (2020): 232237.

[6] Leduc, G., Kubler, S., \& Georges, J. P. (2021). Innovative blockchain-based farming marketplace and smart contract performance evaluation. Journal of Cleaner Production, 306, 127055.

[7] Raj, Jennifer S. "Improved Response Time and Energy Management for Mobile Cloud Computing Using Computational Offloading." Journal of ISMAC 2, no. 01 (2020): 38-49.

[8] Saurabh, S., \& Dey, K. (2021). Blockchain technology adoption, architecture, and sustainable agri-food supply chains. Journal of Cleaner Production, 284, 124731.

[9] Shrestha, Sujan, and Subarna Shakya. "A Comparative Performance Analysis of FogBased Smart Surveillance System." Journal of trends in Compu ter Science and Smart technology (TCSST) 202 (2020): 78-88

[10] Miloudi, L., Rezeg, K., Kazar, O., \& Miloudi, M. K. (2019, July). Smart Sustainable Farming Management Using Integrated Approach of IoT, Blockchain \& Geospatial Technologies. In International Conference on Advanced Intelligent Systems for Sustainable Development (pp. 340-347). Springer, Cham. 
[11] Smys, S., and G. Ranganathan. "Performance Evaluation of Game Theory Based Efficient Task Scheduling For Edge Computing." Journal of ISMAC 2, no. 01 (2020): 50-61.

[12] Verma, M. (2021). Smart contract model for trust based agriculture using blockchain technology. International journal of research and analytical reviews, 8(2), 354-355.

[13] Mkrttchian, V. (2021). Artificial and Natural Intelligence Techniques as IoP-and IoTBased Technologies for Sustainable Farming and Smart Agriculture. In Artificial Intelligence and IoT-Based Technologies for Sustainable Farming and Smart Agriculture (pp. 40-53). IGI Global.

[14] Vivekanadam, B. (2020). Analysis of Recent Trend and Applications in Block Chain Technology. Journal of ISMAC, 2(04), 200-206.

[15] Sivaganesan, D. (2019). Block chain enabled internet of things. Journal of Information Technology, 1(01), 1-8.

[16] Putri, A. N., Hariadi, M., \& Wibawa, A. D. (2020, March). Smart Agriculture Using Supply Chain Management Based On Hyperledger Blockchain. In IOP Conference Series: Earth and Environmental Science (Vol. 466, No. 1, p. 012007). IOP Publishing.

[17] Suma, V. (2019). Security and privacy mechanism using blockchain. Journal of Ubiquitous Computing and Communication Technologies (UCCT), 1(01), 45-54.

[18] Bhalaji, N. (2020). Reliable Data Transmission with Heightened Confidentiality and Integrity in IOT Empowered Mobile Networks. Journal of ISMAC, 2(02), 106-117.

[19] Sivaganesan, D. (2019). Design and development ai-enabled edge computing for intelligent-iot applications. Journal of trends in Computer Science and Smart technology (TCSST), 1(02), 84-94. 